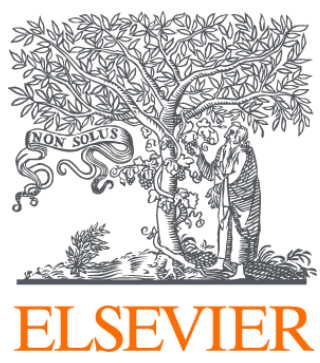

Since January 2020 Elsevier has created a COVID-19 resource centre with free information in English and Mandarin on the novel coronavirus COVID-

19. The COVID-19 resource centre is hosted on Elsevier Connect, the company's public news and information website.

Elsevier hereby grants permission to make all its COVID-19-related research that is available on the COVID-19 resource centre - including this research content - immediately available in PubMed Central and other publicly funded repositories, such as the WHO COVID database with rights for unrestricted research re-use and analyses in any form or by any means with acknowledgement of the original source. These permissions are granted for free by Elsevier for as long as the COVID-19 resource centre remains active. 


\title{
Comparing COVID-19 vaccine allocation strategies in India: A mathematical modelling study
}

\author{
Brody H. Foy ${ }^{\mathrm{a}, \mathrm{b}, 1}$, Brian Wahl ${ }^{\mathrm{c}, \mathrm{d}, 1}$, Kayur Mehta ${ }^{\mathrm{c}, \mathrm{d}}$, Anita Shet ${ }^{\mathrm{c}, \mathrm{d}}$, Gautam I. Menon ${ }^{\mathrm{e}, \mathrm{f}}$, \\ Carl Britto ${ }^{\mathrm{g}, \mathrm{h}, *}$ \\ a Systems Biology Department, Harvard Medical School, USA \\ b Center for Systems Biology and Department of Pathology, Massachusetts General Hospital, USA \\ ${ }^{\mathrm{c}}$ Department of International Health, Johns Hopkins Bloomberg School of Public Health, Baltimore, USA \\ d International Vaccine Access Center, Johns Hopkins Bloomberg School of Public Health, Baltimore, USA \\ e Departments of Physics and Biology, Ashoka University, Sonepat, India \\ ${ }^{\mathrm{f}}$ Theoretical Physics and Computational Biology, The Institute of Mathematical Sciences, Chennai, India \\ ${ }^{\mathrm{g}}$ Department of Pediatrics, Boston Children's Hospital, Boston, MA, USA \\ ${ }^{\mathrm{h}}$ Division of Infectious Disease, St. John's Research Institute, Bengaluru, India
}

A R T I C LE I N F O

\section{Article history:}

Received 22 November 2020

Received in revised form 22 December 2020

Accepted 26 December 2020

\section{Keywords:}

COVID-19

Mathematical modelling

SEIR

Immunization

\begin{abstract}
A B S T R A C T
Background: The development and widespread use of an effective SARS-CoV-2 vaccine could prevent substantial morbidity and mortality associated with COVID-19 and mitigate the secondary effects associated with non-pharmaceutical interventions.

Methods: We used an age-structured, expanded SEIR model with social contact matrices to assess agespecific vaccine allocation strategies in India. We used state-specific age structures and disease transmission coefficients estimated from confirmed incident cases of COVID-19 between 1 July and 31 August 2020. Simulations were used to investigate the relative reduction in mortality and morbidity of vaccine allocation strategies based on prioritizing different age groups, and the interactions of these strategies with concurrent non-pharmaceutical interventions. Given the uncertainty associated with COVID-19 vaccine development, we varied vaccine characteristics in the modelling simulations.

Results: Prioritizing COVID-19 vaccine allocation for older populations (i.e., $>60$ years) led to the greatest relative reduction in deaths, regardless of vaccine efficacy, control measures, rollout speed, or immunity dynamics. Preferential vaccination of this group often produced relatively higher total symptomatic infections and more pronounced estimates of peak incidence than other assessed strategies. Vaccine efficacy, immunity type, target coverage, and rollout speed significantly influenced overall strategy effectiveness, with the time taken to reach target coverage significantly affecting the relative mortality benefit comparative to no vaccination.

Conclusions: Our findings support global recommendations to prioritize COVID-19 vaccine allocation for older age groups. Relative differences between allocation strategies were reduced as the speed of vaccine rollout was increased. Optimal vaccine allocation strategies will depend on vaccine characteristics, strength of concurrent non-pharmaceutical interventions, and region-specific goals.

(c) 2021 The Authors. Published by Elsevier Ltd on behalf of International Society for Infectious Diseases. This is an open access article under the CC BY-NC-ND license (http://creativecommons.org/licenses/by-nc-
\end{abstract}

\section{Introduction}

After first emerging in Wuhan, China in late 2019,(Li et al., 2020) severe acute respiratory syndrome coronavirus 2 (SARS-CoV-2), the virus that causes coronavirus disease 2019 (COVID-19), has

\footnotetext{
* Corresponding author at: Department of Pediatrics, 300 Longwood Avenue, Boston Children's Hospital, Boston, MA 02115, USA.

E-mail address: carl.britto@childrens.harvard.edu (C. Britto).

1 Authors contributed equally.
}

spread rapidly throughout the world causing local epidemics in virtually all countries (Dong et al., 2020). While early, large-scale COVID-19 epidemics occurred primarily in high-income countries in Europe and North America, a significant number of cases and deaths have also now occurred in low- and middle-income countries (Dong et al., 2020). Approximately 1.4 billion people are at risk of SARS-CoV-2 infection in India with many having risk factors for severe outcomes (Nandi et al., 2020).

The first confirmed case of COVID-19 in India was identified in Kerala state on 30 January (Perappadan, 2020). During weeks that followed, several travel-associated cases were confirmed 
throughout the country (Rawat, 2020). To slow the spread of the virus and reduce peak incidence, the central government initiated one of the largest lockdowns in the world on 25 March 2020 comprising non-pharmaceutical interventions (NPIs), including physical distancing and restrictions on non-essential travel (Pulla, 2020). Several analyses indicate that lockdown measures substantially reduced the effective reproduction number $\left(R_{t}\right)$ in the country (Gupta et al., 2020; Kumar et al., 2020; Sardar et al., 2020). However, the lockdown precipitated several secondary effects, including reduced livelihoods, difficulty accessing routine health services, and mass migrations (The Lancet, 2020). Lockdown measures were relaxed beginning on 1 June 2020. As of 12 November 2020, India has reported approximately 8.6 million cases and 130,000 deaths (Dong et al., 2020).

In the absence of a highly effective therapeutic agent for COVID19 , the development of vaccines that provide protection from SARS-CoV-2 infection is a global imperative. An unprecedented effort is currently underway to rapidly develop effective COVID-19 vaccines, with several stakeholders working together to condense the process into months (Graham, 2020). Of the numerous vaccine candidates under development, 11 are in Phase III clinical trials as of November 2020 (Milken Institute, 2020). Five COVID-19 vaccines have been approved for limited use; however, Phase III clinical trial data are not yet available for these vaccines and they have not been prequalified by the World Health Organization (WHO) (Milken Institute, 2020). In India, clinical development is currently underway for multiple candidate vaccines.

New mechanisms are also being established to expedite manufacturing and deployment and support the fair distribution of COVID-19 vaccines (World Health Organization, 2020a). The COVAX Facility is a global risk-sharing mechanism for the pooled procurement of COVID-19 vaccines. Through this mechanism, 92 low- and middle-income countries, including India, are eligible to be supported by the COVAX Advance Market Commitment (AMC), which will pay for the cost of COVID-19 vaccines once COVID-19 vaccines have been licensed and prequalified by the World Health Organization (2020a). Countries participating in the COVAX Facility are encouraged to vaccinate frontline health workers and social care workers first (World Health Organization, 2020b). As the supply of COVID-19 vaccines increases, the distribution of COVID-19 vaccines through the COVAX Facility will continue such that $20 \%$ of country populations can be covered, specifically those at increased risk.

In the context of limited supply and to support policies related to COVID-19 vaccine allocation in India, we developed a mathematical model to simulate different vaccine allocation strategies. There remain several unknowns associated with the current COVID-19 vaccine development. Therefore, we assessed these vaccine allocation strategies varying potential vaccine characteristics. We also evaluated the relative reduction in cases and deaths under of varying control measures. The findings of this analysis could also be used by other low- and middle-income countries to inform their COVID-19 vaccine allocation strategies.

\section{Methods}

Data collection

Daily and state-specific confirmed incident SARS-CoV-2 infection case data were collected from multiple sources, including the Ministry of Health and Family Welfare, the Indian Council of Medical Research, and a website for crowd-sourced information related to COVID-19 (www.covid19india.com). The data available from this website are collated from public sources and validated by a group of volunteers.

\section{Model of disease transmission}

Disease transmission was modelled using an age-structured compartment model, stratified into ten-year age bands $(0-10,10-$ $20,[\ldots], 60-70, \geq 70$ years). The model includes different compartments for each age band and infection state (i.e., $S, E, A$, $I, Q$, and $R$ ). We assume subjects start susceptible to infection $(S)$ and can become exposed $(E)$ after contact with an infectious individual. After a latent period, exposed subjects either develop an asymptomatic $(A)$ or symptomatic $(I)$ infection, with an agestratified probability. Subjects with symptomatic infections are hospitalized or choose to self-isolate $(Q)$ at a given rate. Once hospitalized or isolated, subjects either recover $(R)$ or die $(D)$, with an age-stratified mortality rate. Asymptomatic individuals are assumed to have no risk of mortality and simply recover at a given rate. Recovered subjects are assumed to become susceptible at a given rate, reflecting eventual loss of temporary immunity from the infection (Sariol and Perlman, 2020). We assumed that COVID19 vaccines are allocated gradually into a specific age-defined community at a constant rate.

We simulated two different mechanism through which COVID19 vaccines could induce immunity (Peiris and Leung, 2020). In one simulation, vaccinated individuals $(V)$ are protected from infection and therefore unable to infect others (i.e., sterilizing immunity). In the other simulation, vaccinated individuals are not protected from asymptomatic infection and therefore can infect others if they become infected (i.e., non-sterilizing immunity). In the latter, if an individual develops an asymptomatic infection after receiving a vaccine that does not confer sterilizing immunity, they are assumed to have a temporary immunity from developing further asymptomatic infections, with immunity waning at the same rate as non-vaccinated subjects who recover from infection. Formulated as a system of differential equations, and using $S_{i}$ to denote the susceptible population from age group $i$, for each age group our model comprises:

$$
\begin{aligned}
& \frac{d S_{i}}{d t}=\mu R_{i}-\varepsilon M-\frac{\beta_{1}}{N} \sum_{j=1}^{K} C_{i j} S_{i}\left(A_{j}+A_{j}^{v}+I_{j}\right), \\
& \frac{d V_{i}}{d t}=\mu R_{i}^{v}+\varepsilon M-\frac{\beta_{2}}{N} \sum_{j=1}^{K} C_{i j} V_{i}\left(A_{j}+A_{j}^{v}+I_{j}\right), \\
& \frac{d E_{i}}{d t}=-\sigma E_{i}+\frac{\beta_{1}}{N} \sum_{j=1}^{K} C_{i j} S_{i}\left(A_{j}+A_{j}^{v}+I_{j}\right), \\
& \frac{d E_{i}^{v}}{d t}=-\sigma E_{i}^{v}+\frac{\beta_{2}}{N} \sum_{j=1}^{K} C_{i j} V_{i}\left(A_{j}+A_{j}^{v}+I_{j}\right)
\end{aligned}
$$$$
\frac{d A_{i}}{d t}=\left(1-p_{i}\right) \sigma E_{i}-\gamma A_{i}
$$

$$
\frac{d A_{i}^{v}}{d t}=\sigma E_{i}^{v}-\gamma A_{i}^{v}
$$$$
\frac{d I_{i}}{d t}=p_{i} \sigma E_{i}-\alpha I_{i}
$$ 
$\frac{d Q_{i}}{d t}=\alpha I_{i}-\omega Q_{i}$

$\frac{d R_{i}}{d t}=\gamma A_{i}+\left(1-\delta_{i}\right) \omega Q_{i}-\mu R_{i}$

$\frac{d R_{i}^{v}}{d t}=\gamma A_{i}^{v}-\mu R_{i}^{v}$

$\frac{d D_{i}}{d t}=\delta_{i} \gamma_{2} Q_{i}$

where $1 / \mu$ is the average length of immunity, $\beta_{1}$ is the force of infection, $N$ is the total population size, $1 / \sigma$ is the average latent period, $p$ is the proportion of infections which are symptomatic, $1 / \gamma$ is the average asymptomatic recovery time, $1 / \omega$ is the average time from isolation to recovery for a symptomatic infection, $1 / \alpha$ is the average time until a symptomatically infectious subject selfquarantines or is hospitalized, and $\delta$ is the likelihood of death given symptomatic infection. $C_{i j}$ is the relative frequency of contact between age group $i$ and age group $j$. For the simulation where vaccines confer non-sterilizing immunity, $\beta_{2}=\beta_{1}$ and $E^{V}, A^{V}$, and $R^{V}$ denote subjects who are exposed, asymptomatic, and recovered, respectively. For the simulation where COVID-19 vaccines provide sterilizing immunity, $\beta_{2}=0$, meaning those who are vaccinated cannot become infectious. In both cases, vaccines are assumed to be rolled out gradually, with $M$ doses available each day, and an efficacy of $\varepsilon$. We assumed that subjects moved to the vaccinated $(V)$ compartment only once they received all doses of the COVID-19 vaccine. Figure 1 is flow diagram of transitions within the model.

Contact matrices $(C)$ were estimated from social mixing patterns in the Indian population (Prem et al., 2017). Estimates were broken down into four categories, representing the mixing patterns in different environments: (1) "at home", (2) "at school", (3) "at work", and (4) "other"; with $C$ representing the summation of the mixing matrices. In normal scenarios (i.e., no control measures), each mixing pattern was equally weighted. Under strong control measures weights of $1.21,0.56,0.0$, and 0.45 were used for "at home", "at work", "at school", and "other" matrices, respectively, based on estimates from Google's mobility data during the lockdown period (March 25-May 31, 2020) (Aktay et al., 2020). Moderate control measures were simulated using the average between no control and strong control measure weights.

All parameters except $\beta$ were estimated based on prior studies, with a full list of parameters and their sources given in Table 1. $\beta$ values were estimated based on fits of the model-simulated, hospitalized or self-isolated population numbers $(Q)$ against confirmed active infection case numbers, between 1 June and 31 July (i.e., after the lockdown period). Given variability in social mixing patterns immediately after the national lockdown, $\beta$ values were estimated assuming moderate control measures and no control measures during this period. Similar fits were obtained when fitting model-simulated deaths $(D)$ against reported deaths and when simultaneously fitting $D$ and $Q$ against infection and death case numbers (supplemental material). Parameter fitting was performed using MATLAB's Statistical Toolbox with an example data fit presented in the supplemental materials. All models were simulated in MATLAB and use forward Euler discretisation for the differential equations, with a timestep of one day.

\section{Vaccination strategies}

Four age-based vaccination strategies were considered: (1) vaccines are distributed evenly across the entire population or were first distributed to those who were: (2) 20-40 years, (3) 4060 years, or $(4) \geq 60$ years. In strategies $2-4$, following vaccination of the target age group to the assumed vaccine coverage, vaccine doses were allocated to the remaining population proportional to the size of the remaining age-groups. Simulations were performed using a range of vaccine efficacies and assuming a fixed number of doses available each day.

Within this framework, simulations were performed using efficacy, age-specific population coverage ranging from 0 to $100 \%$ and considering vaccines that provide sterilizing and nonsterilizing immunity. Dose availability was assumed constant over time, reflecting the market pressures of acquiring vaccine doses,

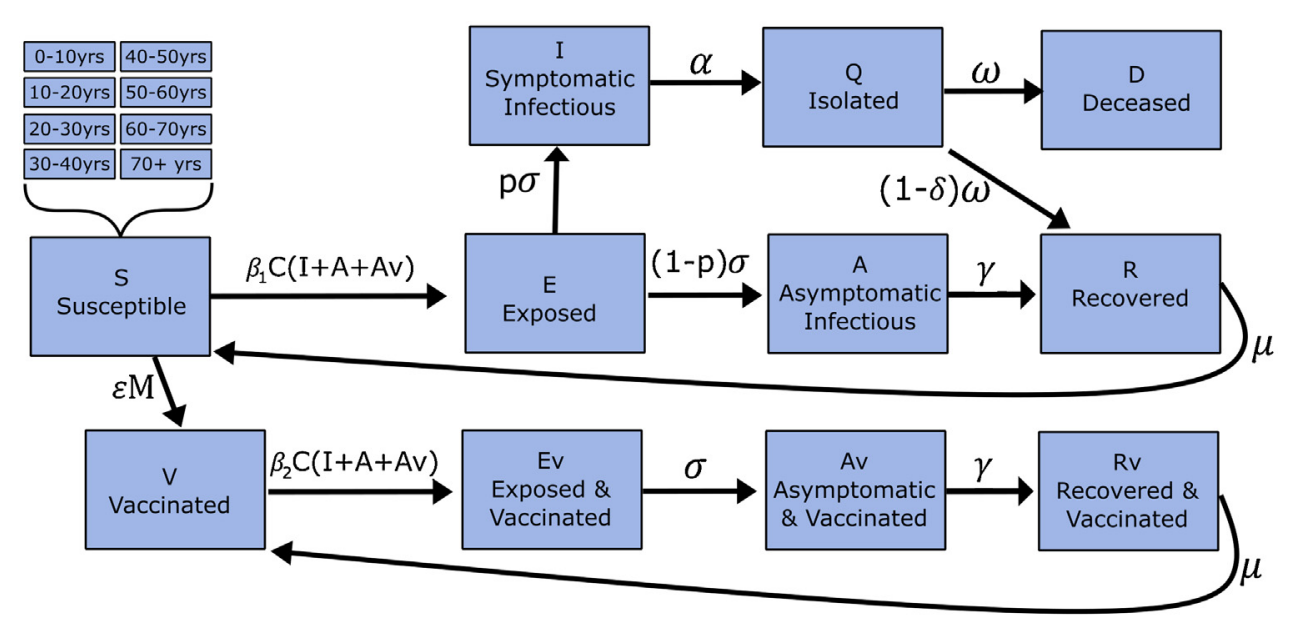

Figure 1. Schematic of model transmission dynamics.

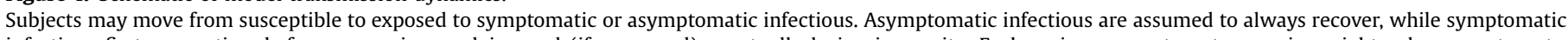

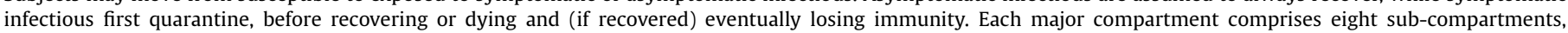

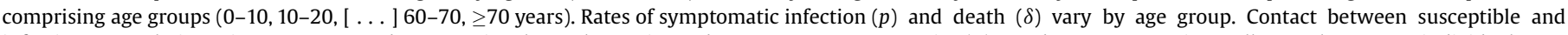

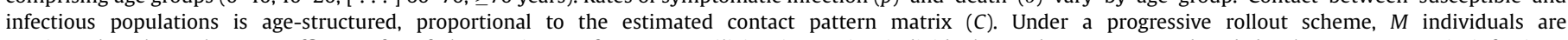

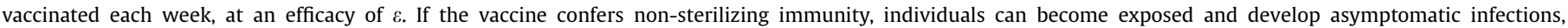

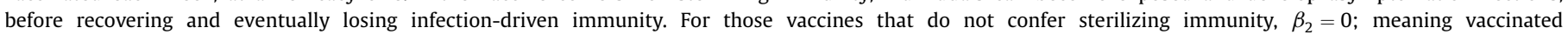
individuals no longer contribute to transmission dynamics. 
Table 1

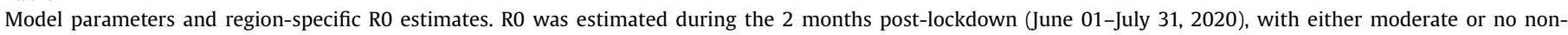
pharmaceutical interventions.

\begin{tabular}{|c|c|c|c|c|c|}
\hline \multicolumn{4}{|l|}{$R_{0}$ Estimates } & \multirow{2}{*}{\multicolumn{2}{|c|}{ Value (moderate NPIs }} \\
\hline Region & Population (millions) & & Value (no NPIs) & & \\
\hline Uttar Pradesh & 204.2 & & 2.41 & & 3.22 \\
\hline Maharashtra & 114.0 & & 2.20 & & 2.89 \\
\hline Bihar & 104.1 & & 2.36 & & 3.15 \\
\hline West Bengal & 90.3 & & 2.33 & & 3.10 \\
\hline Madhya Pradesh & 72.6 & & 1.81 & & 2.39 \\
\hline Tamil Nadu & 72.1 & & 2.31 & & 3.12 \\
\hline Rajasthan & 68.5 & & 2.00 & & 2.59 \\
\hline Karnataka & 64.1 & & 3.74 & & 4.95 \\
\hline Gujarat & 60.4 & & 1.89 & & 2.49 \\
\hline Andhra Pradesh & 49.5 & & 2.93 & & 3.92 \\
\hline \multicolumn{6}{|l|}{ Other model parameters } \\
\hline \multicolumn{2}{|l|}{ Parameter } & \multicolumn{2}{|l|}{ Value } & \multicolumn{2}{|l|}{ Reference } \\
\hline \multirow{7}{*}{\multicolumn{2}{|c|}{$\begin{array}{l}1 / \sigma \text { (latent period) } \\
1 / \gamma \text { (recovery period) } \\
1 / \alpha \text { (pre-isolation infection period) } \\
1 / \omega \text { (post-isolation recovery period) } \\
1 / \mu \text { (immunity duration) } \\
\mathrm{p} \text { (proportion of symptomatic infections) } \\
\Delta \text { (case fatality rate) }\end{array}$}} & \multicolumn{2}{|l|}{5.1 days } & \multicolumn{2}{|l|}{ Lauer et al. (2020) } \\
\hline & & \multicolumn{2}{|l|}{21 days } & \multicolumn{2}{|l|}{ Bi et al. (2020) } \\
\hline & & \multicolumn{2}{|l|}{4.6 days } & \multicolumn{2}{|l|}{ Bi et al. (2020) } \\
\hline & & \multicolumn{2}{|l|}{16.4 days } & \\
\hline & & \multicolumn{2}{|l|}{1 year } & \multicolumn{2}{|c|}{ Estimated (other values in supplemental material) } \\
\hline & & \multicolumn{2}{|l|}{ Age-specific } & \multicolumn{2}{|c|}{ Davies et al. (2020) } \\
\hline & & Age-specific & & \multicolumn{2}{|l|}{ Laxminarayan et al. (2020) } \\
\hline
\end{tabular}

and the logistic pressures of distributing these doses. Dose availability was expressed as the percentage of the population which could be vaccinated each month, with simulations using values from 2 to 15\%, reflecting an approximate time of between six months to four years to vaccinate to $100 \%$ of the target population. Results are presented over a five-year period, reflecting an approximate upper bound on the likely time to achieve target vaccine coverage (i.e., target coverage can be achieved in less time). Nevertheless, given potential changes in vaccine availability, we also present results over a one-year time period for comparison (supplemental material).

\section{Results}

\section{Parameter estimates}

$R_{0}$ values were estimated for the 10 most populous states within India, assuming moderate control measures and no control measures. Estimates are given in Table 1 , with mean $R_{0}$ values of 2.4 assuming no control measures and 3.2 assuming moderate control measures during the lockdown period. We used the former as the base case value in our simulations. Minimum and maximum values were 1.8 and 5.0 respectively, with results using these values in the supplemental materials. The values in Table 1 are consistent with $R_{0}$ values reported for other countries (Gatto et al., 2020; Sanche et al., 2020; Wu et al., 2020; Zhao et al., 2020). Within our model, the implementation of moderate and severe control measures led to a $23 \%$ and $44 \%$ relative reduction in $R_{0}$, respectively.

\section{Vaccine strategy simulations}

Four vaccine strategies were simulated under variations in dosage availability, target group coverage, vaccine efficacy, effect on transmission (i.e., sterilizing or non-sterilizing immunity), and the implementation of other control measures (i.e., no lockdown, moderate lockdown, or strong lockdown). Example epidemic curves for COVID-19 vaccines that confer sterilizing and nonsterilizing immunity are given in Figure 2. Regardless of vaccination strategy and immunization coverage in the target population, the initial infection wave occurs at a similar time, though with varying severity based on strategy. However, COVID-19 vaccines that confer sterilizing immunity appear to minimize the extent of future infection waves. In both cases strategy 4 (i.e., prioritizing individual $\geq 60$ years) leads to the greatest reduction in deaths; however, all vaccination strategies produce significant benefits comparative to no vaccination.

Within Figure 3, we present the estimated reduction in deaths and symptomatic infections over a five-year period using each of the four vaccination strategies, under varying efficacy, control measures, and rollout speeds. All results are presented relative to the outcomes with no vaccination, using the same $R_{0}$ value, and with no control measures. Simulations were performed using an $R_{0}$ of 2.4 (i.e., the mean $R_{0}$ value in 10 states) and assume a target COVID-19 vaccine coverage of $75 \%$. Results in Figure 3 illustrate that prioritizing vaccine allocation among older adults consistently results in the greatest reduction in deaths, regardless of vaccine efficacy, control measures, rollout speed, or immunity type. Conversely, all four strategies result in extremely similar reductions in symptomatic infection rates, with the optimal strategy being dependent on the specific implementation and vaccine. The relative benefit of prioritizing vaccine allocation among older adults compared to other strategies is highest under slower rollout speeds, while overall benefit is greatest the faster the rollout speed.

Overall reduction in deaths is strongly limited by vaccine efficacy, and is strongly influenced by control measures, with more severe measures leading to greater reductions. Similar patterns were seen with different $R_{0}$ values, target coverages, and immunity assumptions (supplemental materials). Similar patterns were also seen when assuming imperfect self-isolation of symptomatically infectious individuals (supplemental materials), and when assuming some degree of disease transmission by pre-symptomatic individuals (supplemental materials).

While $R_{0}$ values, vaccine efficacy, and other vaccine characteristics (i.e., sterilizing versus non-sterilizing immunity) all influence strategy effectiveness, in application these factors are immutable from the perspective of policy makers. Rather, international and national efforts, including investments and policies, can primarily influence three factors: (1) dosage availability/rollout speed, (2) target vaccine coverage; and (3) the continuation or relaxation of control measures. Within this context, in Figure 4 we present the relative reduction in deaths for vaccine allocation prioritizing older adults as each of those factors is modified. Equivalent results for 

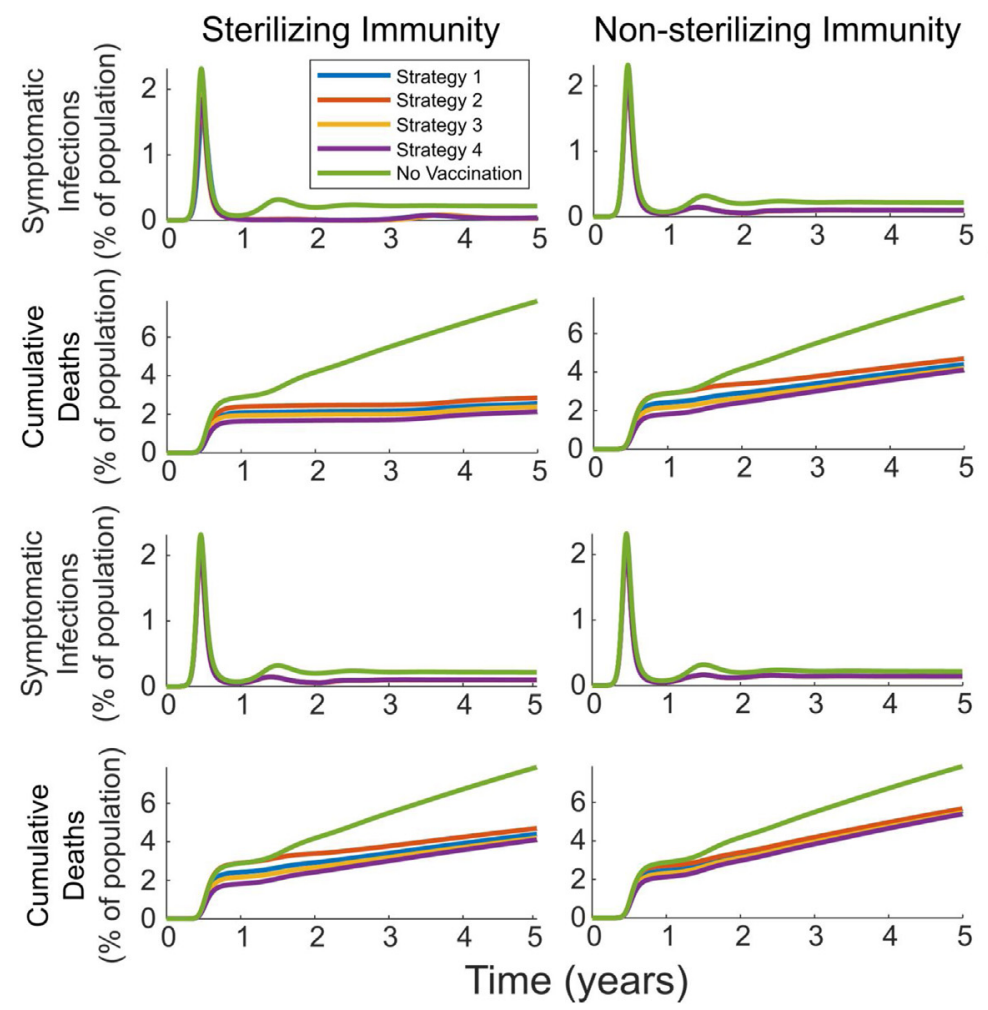

\section{$50 \%$ Coverage}

Figure 2. Simulated infection curves and cumulative deaths with four vaccination strategies.

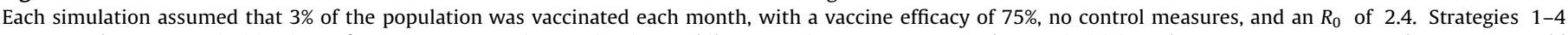

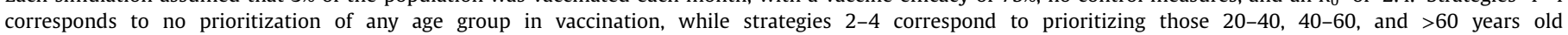
respectively. Strategy 4 leads to the greatest reduction in deaths, though all strategies perform better than no vaccination.

strategies 1-3 are presented in the supplemental materials. Under low coverage, the speed at which the vaccine is rolled out has little effect on the overall reduction in deaths.

\section{Discussion}

Our findings support international recommendations to prioritize COVID-19 vaccine allocation for older adults (World Health Organization, 2020b), as it contributed to the greatest relative reduction in overall mortality in all scenarios considered. Our analyses indicate that prioritising younger populations will have a greater impact on reducing incidence of infections relative to prioritizing older age groups. However, these reductions are marginal and prioritizing younger age groups will contribute the lowest relative reduction on COVID-19 mortality compared to other strategies, including equal distribution to the general population. These findings were consistent, although to different degrees, across all model iterations, including COVID-19 vaccines that confer sterilizing and non-sterilizing immunity. A similar framework for equitable allocation of COVID-19 vaccine that prioritised older populations was adopted by the panel of experts from the Centers for Disease Control and Prevention (CDC), the National Institutes of Health (NIH) and the National Academies (Gayle et al., 2020).

The characteristics of first-generation COVID-19 vaccines remain a question of debate (Peiris and Leung, 2020). However, it is unlikely that the first vaccines will provide $100 \%$ protective efficacy nor protection from asymptomatic carriage. Most candidate vaccines that are currently in Phase III trials aim to assess efficacy against clinical disease (Peiris and Leung, 2020). Recent reports from leading COVID-19 vaccine candidates in advanced clinical development indicate vaccine efficacy against confirmed cases of $>90 \%$, including among older populations (Callaway, 2020). The WHO has indicated that a successful vaccine should be $50 \%$ efficacious (Krause et al., 2020). We observed greater differences between COVID-19 vaccine allocation strategies at higher vaccine efficacy values for relative reductions in deaths. Vaccines that confer sterilizing immunity also led to greater relative reductions of cases and deaths compared to vaccine that did not provide sterilizing immunity. This is likely attributable to the fact that sterilizing vaccines disrupt viral transmission of. However, COVID-19 vaccine challenge studies in primates demonstrated reductions in symptomatic disease and viral load, but did not produce sterilising immunity (Corbett et al., 2020; van Doremalen et al., 2020).

Policy makers around the world, especially those in low- and middle-income countries, have had to make difficult decisions related to the implementation and relaxation of lockdown measures. Lockdown measures help to reduce transmission of the virus but have been associated with several

secondary effects, including reduced livelihoods (Walker et al., 2020), increased morbidity and mortality due to limited utilization of routine health services (Roberton et al., 2020), and several psychosocial and mental health implications (Roy et al., 2020). Effective COVID-19 vaccines could alleviate the need for restrictive lockdown measures. Our model allowed us to make relative comparisons of COVID-19 vaccine allocation strategies in the context of various control measures. We found that the relative reduction in cases and deaths does not meaningfully change based on the level or absence of control measures when the vaccine does not provide sterilizing immunity. However, in the model where effective vaccines do provide sterilizing immunity, the relative 

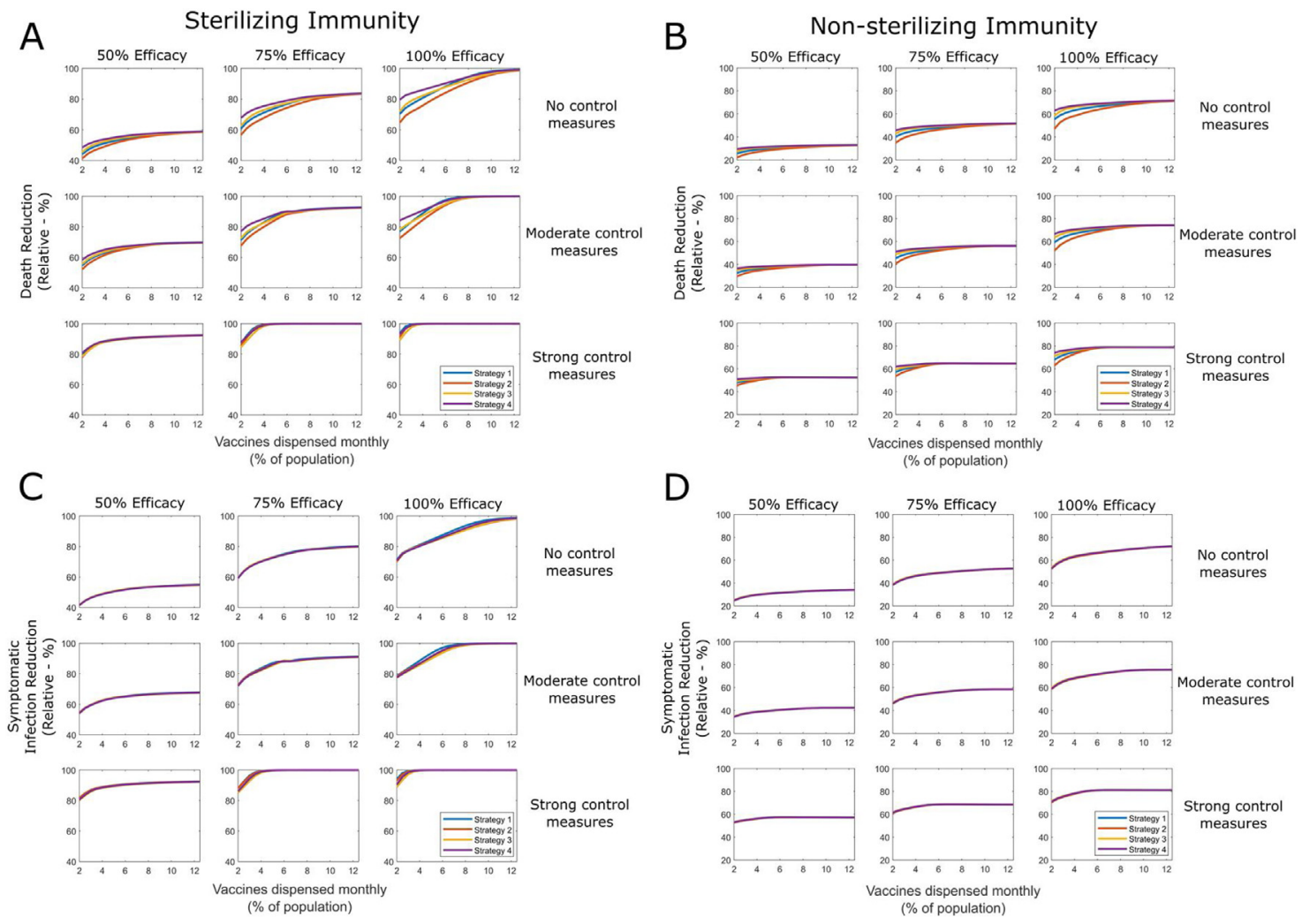

Figure 3. Comparison of benefit for four different vaccination strategies, against no vaccination.

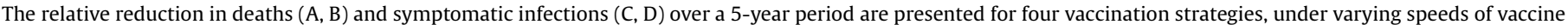

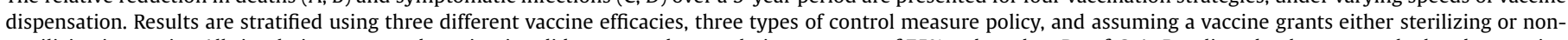

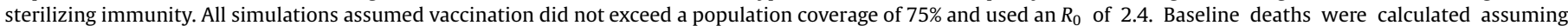
no control measures and the same Ro value.

reduction in cases and deaths is substantially greater when strong control measures are in place.

In accordance with COVAX Facility requirements (World Health Organization, 2020a), the allocation of COVID-19 vaccines to health care workers and social workers should be prioritized. There remains an acute health workforce shortage in many parts of India (Rao et al., 2016; Shrivastava and Shrivastava, 2019). Immunizing this important population with priority, together with adequate supply of personal protective equipment, will help to strengthen the resiliency of the fragile health system during the epidemic. While health workers remain at higher risk of SARS-CoV-2 infection (Nguyen et al., 2020), there is insufficient evidence to determine how they contribute to transmission. Our model did not consider a health worker compartment.

India has a robust national immunization program for early childhood that has been strengthened recently with demonstrable gains in vaccination coverage (Gurnani et al., 2018). The recent introduction and rollout of the pneumococcal conjugate vaccine and rotavirus vaccine have shown that new vaccines can be successfully rolled out within existing public health infrastructure (Malik et al., 2019). While a clear strategy for childhood vaccination exists globally and in India, a blueprint for adult immunization is recognizably inadequate and is being increasingly acknowledged as important for sustaining and enhancing health outcomes (Privor-Dumm et al., 2020). So far, India has initiated the process of targeting adults by setting up of health centers adult and immunization as an example of a life course approach to health services (Lahariya and Bhardwaj, 2020).

During the influenza pandemic of 2009, the WHO Initiative was able to deploy almost 80 million doses of pandemic H1N1 vaccine to resource-limited settings in 77 poorest countries (World Health Organization, 2012). This experiences deploying vaccines in pandemic settings provide lessons that should be utilized to enhance current allocation vaccine plans. First, the availability of robust evidence of demographics, including at-risk population groups is critical for successful vaccine deployment. Simulations with varying scenarios, such the current report, can complement evidence and play an important role in allocation decisions. Second, coordinated planning of national vaccine deployment, including establishment of a robust supply chain management system, was crucial to effective utilization of scarce vaccine resources. Third, funding support from global agencies, local funders, and governments helped sustain vaccine rollout. Finally, public communication and clear messaging was essential to enhancing public confidence in vaccines. Due to data availability constraints, and evolving scientific understanding of COVID-19, the model makes a number of key assumptions about COVID-19 epidemiology and transmission dynamics. Age for confirmed cases and deaths were not available in publicly available data. We therefore used published data from India to inform age-specific dynamics and fatality. Infection was assumed to provide temporary immunity against reinfection for one year, with other values explored in the supplemental materials. The actual average length of immunity due to COVID-19 infection is not precisely known and likely varies based on infection severity (Randolph and Barreiro, 2020; van der Heide, 2020; Wajnberg et al., 2020). Many model parameters, such as force of infection, latent period, time to recovery, and vaccine efficacy all likely vary with age, and potentially with time. However, given lack of clear data, these factors were assumed constant. In addition, due to data availability, 


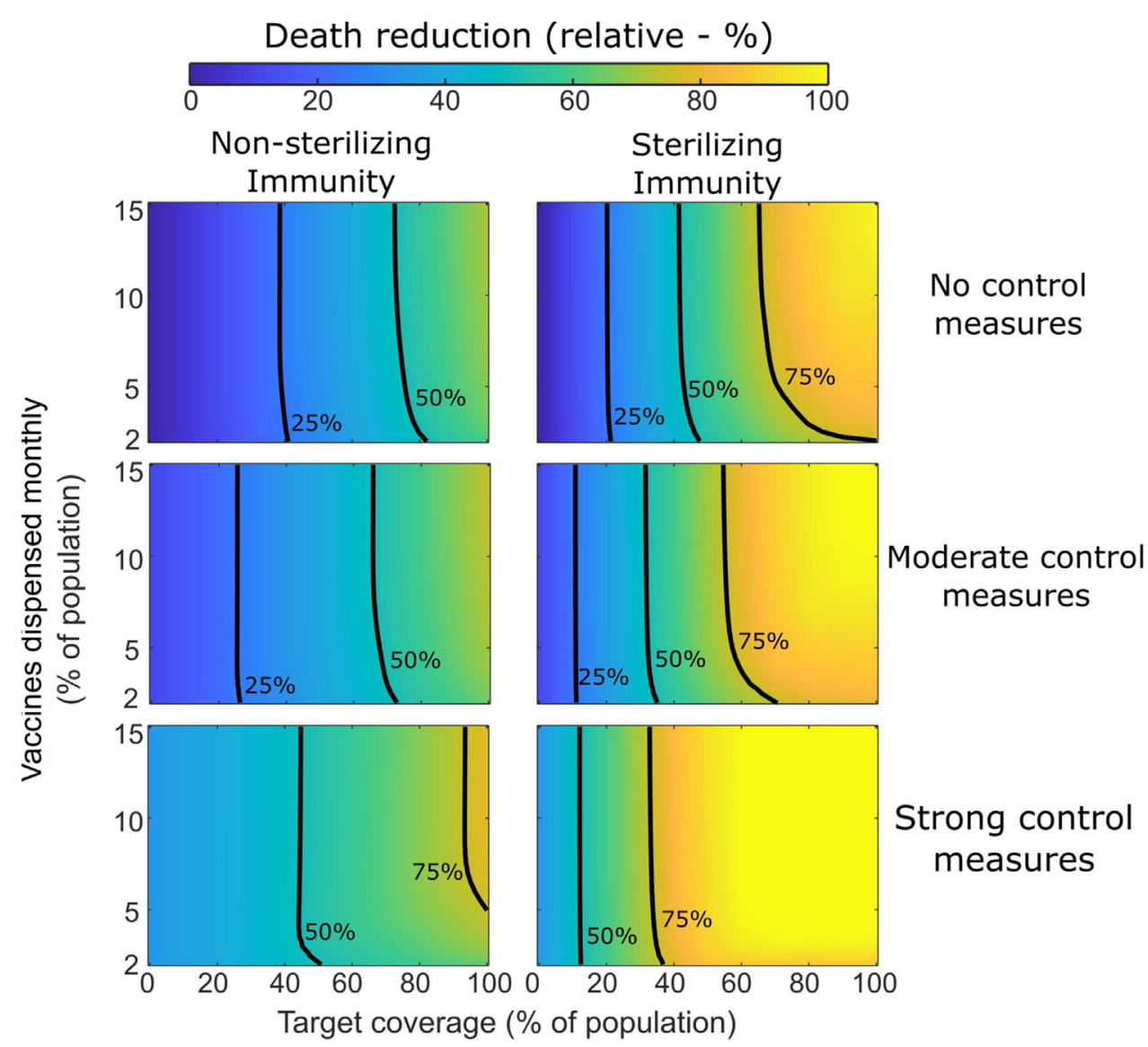

Figure 4. Relative reduction in deaths using vaccination strategy four.

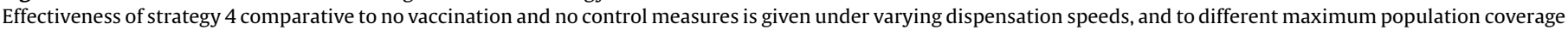

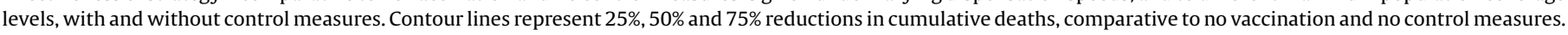
All simulations were performed using an $R_{0}$ of 2.4 .

deaths were estimated using case fatality ratios and not infection fatality ratios, under the assumption that discrepancies between case and infection fatality ratios are predominantly due to undetected asymptomatic infections.

Within the model, vaccines were distributed to a target coverage level, which was constant for each age group. For practical implementation, certain age groups will likely be easier to reach and less reticent to vaccination than others, meaning true coverage may vary by age (Cobos Muñoz et al., 2015). Preliminary evidence suggests that COVID-19 may be subject to seasonal forcing (Sajadi et al., 2020). This was not accounted for in the model to lack of a clear timeline for when vaccine rollout would begin. Given current understanding of COVID-19 immunity dynamic, there will likely be some prevalence of infection-driven immunity that exists before vaccine rollout begins. However, given uncertainties associated with vaccine delivery timelines, expected seroprevalence estimates, and the quality and duration of immunity from natural infection, there is no reliable data to inform this within the model. As a result, no prior immunity within the population was assumed. More broadly, this model was designed for comparison between vaccination strategies, and is not meant provide exact estimates of cumulative deaths or symptomatic infections. Rather results are meant to represent the estimated relative benefit of different scenarios.

\section{Conclusions}

Progress towards development and approval of SARS-CoV-2 vaccines has been extraordinarily fast; however, challenges of fair and optimal allocation remain. Supply limitations and logistic challenges suggest that vaccine administration across India will be slow, necessitating distribution strategies that offer the greatest protection. We illustrate that when accounting for Indian population structure, vaccination of older age groups ( $>60$ years) consistently provides the greatest reduction in cumulative deaths. Prioritized vaccination of younger age groups was often seen to reduce symptomatic infection, but this benefit was typically offset by larger case fatality in older populations. Prioritized vaccination of older populations was seen to be optimal regardless of vaccine efficacy, dispensation speed, force of infection, and target coverage, and independent of whether NPIs were implemented.

\section{Conflict of interest}

The authors report no conflicts of interest.

\section{Sources of support}

The authors acknowledge partial funding support from the Johns Hopkins Maternal and Child Health Center - India.

\section{Author contributions}

$\mathrm{CB}$ conceptualized the project and collated the data. BF and BW developed the model and developed the code. BF prepared the visualizations. All authors contributed to the design of the model, the interpretation of the results, and the initial draft of the manuscript. 


\section{Ethical approvals}

No human subjects were involved in this work and therefore ethical approvals were not required for the development of this manuscript.

\section{Appendix A. Supplementary data}

Supplementary material related to this article can be found, in the online version, at doi:https://doi.org/10.1016/j.ijid.2020.12.075.

\section{References}

Aktay A, Bavadekar S, Cossoul G, Davis J, Desfontaines D, Fabrikant A, et al. Google COVID-19 community mobility reports: anonymization process description (version 1.0). arXiv 2020;200404145 preprint arXiv.

Bi Q, Wu Y, Mei S, Ye C, Zou X, Zhang Z, et al. Epidemiology and transmission of COVID-19 in 391 cases and 1286 of their close contacts in Shenzhen, China: a retrospective cohort study. Lancet Infect Dis 2020;, doi:http://dx.doi.org/ 10.1016/S1473-3099(20)30287-5.

Callaway E. COVID Vaccine Excitement Builds as Moderna Reports Third Positive Result. 2020.

Cobos Muñoz D, Monzón Llamas L, Bosch-Capblanch X. Exposing concerns about vaccination in low- and middle-income countries: a systematic review. Int J Public Health 2015;60:767-80, doi:http://dx.doi.org/10.1007/s00038-0150715-6.

Corbett KS, Flynn B, Foulds KE, Francica JR, Boyoglu-Barnum S, Werner AP, et al. Evaluation of the mRNA-1273 vaccine against SARS-CoV-2 in nonhuman primates. N Engl J Med 2020;383:1544-55, doi:http://dx.doi.org/10.1056/ NEJMoa2024671.

Davies NG, Klepac P, Liu Y, Prem K, Jit M. Age-dependent effects in the transmission and control of COVID-19 epidemics. Nat Med 2020;26:1205-11, doi:http://dx. doi.org/10.1038/s41591-020-0962-9.

Dong E, Du H, Gardner L. An interactive web-based dashboard to track COVID-19 in real time. Lancet Infect Dis 2020;20:533-4, doi:http://dx.doi.org/10.1016/ S1473-3099(20)30120-1.

Gatto M, Bertuzzo E, Mari L, Miccoli S, Carraro L, Casagrandi R, et al. Spread and dynamics of the COVID-19 epidemic in Italy. Effects of Emergency Containment Measures Proceedings of the National Academy of Sciences 2020;117:. p. 1048491, doi:http://dx.doi.org/10.1073/pnas.2004978117.

Gayle H, Foege W, Brown L, Kahn B. Framework for Equitable Allocation of COVID-19 Vaccine, 10. Washington, DC: The National Academy Press; 2020. p. 25917.

Graham BS. Rapid COVID-19 vaccine development. Science 2020;368:945-6, doi: http://dx.doi.org/10.1126/science.abb8923.

Gupta M, Mohanta SS, Rao A, Parameswaran GG, Agarwal M, Arora M, et al. Transmission dynamics of the COVID-19 epidemic in India and modelling optimal lockdown exit strategies. medRxiv 2020;, doi:http://dx.doi.org/10.1101/ 2020.05.13.20096826 2020.2005.2013.20096826.

Gurnani V, Haldar P, Aggarwal MK, Das MK, Chauhan A, Murray J, et al. Improving vaccination coverage in India: lessons from Intensified Mission Indradhanush, a cross-sectoral systems strengthening strategy. BMJ (Clinical Research ed) 2018;363:, doi:http://dx.doi.org/10.1136/bmj.k4782 k4782-k4782.

Krause P, Fleming TR, Longini I, Henao-Restrepo AM, Peto R, World Health Organization Solidarity Vaccines Trial Expert Group. COVID-19 vaccine trials should seek worthwhile efficacy. Lancet 2020;396:741-3, doi:http://dx.doi.org/ 10.1016/S0140-6736(20)31821-3.

Kumar R, Malik MZ, Shah SR. Effects of (Un)lockdown on COVID-19 transmission: a mathematical study of different phases in India. medRxiv 2020;, doi:http://dx. doi.org/10.1101/2020.08.19.20177840 2020.2008.2019.20177840.

Lahariya C, Bhardwaj P. Adult vaccination in India: status and the way forward. Hum Vaccin Immunother 2020;16:1508-10, doi:http://dx.doi.org/10.1080/ 21645515.2019.1692564.

Lauer SA, Grantz KH, Bi Q, Jones FK, Zheng Q, Meredith HR, et al. The incubation period of coronavirus disease 2019 (COVID-19) from publicly reported confirmed cases: estimation and application. Ann Intern Med 2020; doi: http://dx.doi.org/10.7326/m20-0504.

Laxminarayan R, Wahl B, Dudala SR, Gopal K, Mohan BC, Neelima S, et al. Epidemiology and transmission dynamics of COVID-19 in two Indian states. Science 2020;, doi:http://dx.doi.org/10.1126/science.abd7672 eabd7672.

Li Q, Guan X, Wu P, Wang X, Zhou L, Tong Y, et al. Early transmission dynamics in Wuhan, China, of novel coronavirus-infected pneumonia. N Engl J Med 2020;382:1199-207, doi:http://dx.doi.org/10.1056/NEJMoa2001316.

Malik A, Haldar P, Ray A, Shet A, Kapuria B, Bhadana S, et al. Introducing rotavirus vaccine in the Universal Immunization Programme in India: from evidence to policy to implementation. Vaccine 2019;37:5817-24, doi:http://dx.doi.org/ 10.1016/j.vaccine.2019.07.104.

Milken Institute. COVID-19 Treatment and Vaccine Tracker. 2020. https://covid19tracker.milkeninstitute.org/\#vaccines intro.

Nandi A, Balasubramanian R, Laxminarayan $\mathrm{R}$. Who is at the highest risk from COVID-19 in India? analysis of health, healthcare access, and socioeconomic indicators at the district level. medRxiv 2020;
Nguyen LH, Drew DA, Graham MS, Joshi AD, Guo CG, Ma W, et al. Risk of COVID-19 among front-line health-care workers and the general community: a prospective cohort study. Lancet Public Health 2020;5:e475-83, doi:http:// dx.doi.org/10.1016/S2468-2667(20)30164-X.

Peiris M, Leung GM. What can we expect from first-generation COVID-19 vaccines?. Lancet 2020;396:1467-9, doi:http://dx.doi.org/10.1016/S0140-6736(20)31976-0.

Perappadan BS. India's First Coronavirus Infection Confirmed in Kerala. New Delhi. 2020.

Prem K, Cook AR, Jit M. Projecting social contact matrices in 152 countries using contact surveys and demographic data. PLoS Comput Biol 2017;13:e1005697, doi:http://dx.doi.org/10.1371/journal.pcbi.1005697.

Privor-Dumm LA, Poland GA, Barratt J, Durrheim DN, Knoll MD, Vasudevan P, et al. A global agenda for older adult immunization in the COVID-19 era: a roadmap for action. Vaccine 2020;, doi:http://dx.doi.org/10.1016/j.vaccine.2020.06.082 S0264-0410X(0220)30885-30889.

Pulla P. Covid-19: India imposes lockdown for 21 days and cases rise. BMJ 2020;368: m1251, doi:http://dx.doi.org/10.1136/bmj.m1251.

Randolph HE, Barreiro LB. Herd immunity: understanding COVID-19. Immunity 2020;52:737-41, doi:http://dx.doi.org/10.1016/j.immuni.2020.04.012.

Rao KD, Shahrawat R, Bhatnagar A. Composition and distribution of the health workforce in India: estimates based on data from the National Sample Survey. WHO South East Asia J Public Health 2016;5:133-40, doi:http://dx.doi.org/ 10.4103/2224-3151.206250.

Rawat M. Coronavirus in India: Tracking Country's First 50 COVID-19 Cases; What Numbers Tell. New Delhi. 2020.

Roberton T, Carter ED, Chou VB, Stegmuller AR, Jackson BD, Tam Y, et al. Early estimates of the indirect effects of the coronavirus pandemic on maternal and child mortality in low-and middle-income countries. Lancet 2020; Available at SSRN 3576549.

Roy A, Singh AK, Mishra S, Chinnadurai A, Mitra A, Bakshi O, et al. Mental health implications of COVID-19 pandemic and its response in India. Int J Soc Psychiatry 2020; $\quad$ doi:http://dx.doi.org/10.1177/0020764020950769 20764020950769-20764020950769.

Sajadi MM, Habibzadeh P, Vintzileos A, Shokouhi S, Miralles-Wilhelm F, Amoros A. Temperature, humidity, and latitude analysis to estimate potential spread and seasonality of coronavirus disease 2019 (COVID-19). JAMA Network Open 2020;3:, doi:http://dx.doi.org/10.1001/jamanetworkopen.2020.11834 e2011834-e2011834.

Sanche S, Lin YT, Xu C, Romero-Severson E, Hengartner N, Ke R. high contagiousness and rapid spread of severe acute respiratory syndrome coronavirus 2. Emerg Infect Dis J 2020;26:1470, doi:http://dx.doi.org/10.3201/eid2607.200282.

Sardar T, Nadim SS, Rana S, Chattopadhyay J. Assessment of lockdown effect in some states and overall India: a predictive mathematical study on COVID-19 outbreak. Chaos Solitons Fractals 2020;139:110078, doi:http://dx.doi.org/ 10.1016/j.chaos.2020.110078.

Sariol A, Perlman S. Lessons for COVID-19 immunity from other coronavirus infections. Immunity 2020;53:248-63, doi:http://dx.doi.org/10.1016/j. immuni.2020.07.005.

Shrivastava SR, Shrivastava PS. Strengthening of the health workforce to meet sustainable development goals in India. J Integr Health Sci 2019;7:30.

The Lancet. COVID-19 in India: the dangers of false optimism. Lancet 2020;396:867, doi:http://dx.doi.org/10.1016/S0140-6736(20)32001-8.

van der Heide V. Neutralizing antibody response in mild COVID-19. Nat Rev Immunol 2020;20:, doi:http://dx.doi.org/10.1038/s41577-020-0325-2 352-352.

van Doremalen N, Lambe T, Spencer A, Belij-Rammerstorfer S, Purushotham J, Port JR, et al. ChAdOx1 nCoV-19 vaccine prevents SARS-CoV-2 pneumonia in rhesus macaques. Nature 2020;586:578-82, doi:http://dx.doi.org/10.1038/s41586020-2608-y.

Wajnberg A, Amanat F, Firpo A, Altman DR, Bailey MJ, Monsour M, et al. Robust neutralizing antibodies to SARS-CoV-2 infection persist for months. Science 2020; , doi:http://dx.doi.org/10.1126/science.abd7728 eabd7728.

Walker PGT, Whittaker C, Watson OJ, Baguelin M, Winskill P, Hamlet A, et al. The impact of COVID-19 and strategies for mitigation and suppression in low- and middle-income countries. Science 2020;369:413-22, doi:http://dx.doi.org/ $10.1126 /$ science.abc0035.

World Health Organization. Main Operational Lessons Learnt From the WHO Pandemic Influenza A(H1N1) Vaccine Deployment Initiative. Geneva: World Health Organization; 2012.

World Health Organization. COVAX: Working for Global Equitable Access to COVID19 Vaccines. 2020. https://www.who.int/initiatives/act-accelerator/covax.

World Health Organization. WHO Concept for Fair Access and Equitable Allocation of COVID-19 Health Products 2020 [October 29, 2020]. 2020.

Wu P, Hao X, Lau EHY, Wong J, Leung KSM, Wu JT, et al. Real-time tentative assessment of the epidemiological characteristics of novel coronavirus infections in Wuhan, China, as at 22 January 2020. Eurosurveillance 2020;25:2000044, ES.2020.25.3.2000044.

Zhao S, Lin Q, Ran J, Musa SS, Yang G, Wang W, et al. Preliminary estimation of the basic reproduction number of novel coronavirus (2019-nCoV) in China, from 2019 to 2020: a data-driven analysis in the early phase of the outbreak. Int J Infect Dis 2020;92:214-7, doi:http://dx.doi.org/10.1016/j.ijid.2020.01.050. 